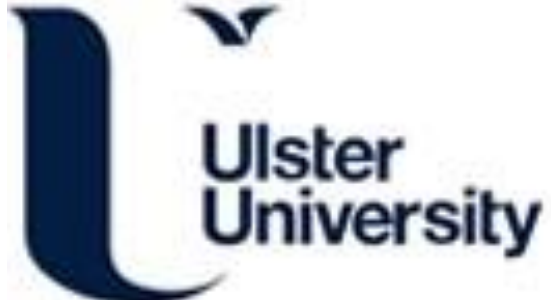

Spatiotemporal Summation of Perimetric Stimuli in Early Glaucoma

Mulholland, P., Redmond, T., Garway-Heath, D., Zlatkova, M., \& Anderson, R. (2015). Spatiotemporal

Summation of Perimetric Stimuli in Early Glaucoma. Investigative Ophthalmology and Visual Science, 56(11),

6473-6482. https://doi.org/10.1167/iovs.15-16921

Link to publication record in Ulster University Research Portal

\section{Published in:}

Investigative Ophthalmology and Visual Science

Publication Status:

Published (in print/issue): 31/10/2015

DOI:

10.1167/iovs.15-16921

\section{Document Version}

Publisher's PDF, also known as Version of record

\section{General rights}

Copyright for the publications made accessible via Ulster University's Research Portal is retained by the author(s) and / or other copyright owners and it is a condition of accessing these publications that users recognise and abide by the legal requirements associated with these rights.

\section{Take down policy}

The Research Portal is Ulster University's institutional repository that provides access to Ulster's research outputs. Every effort has been made to ensure that content in the Research Portal does not infringe any person's rights, or applicable UK laws. If you discover content in the Research Portal that you believe breaches copyright or violates any law, please contact pure-support@ulster.ac.uk. 


\title{
Spatiotemporal Summation of Perimetric Stimuli in Early Glaucoma
}

\author{
Pádraig J. Mulholland, ${ }^{1,2}$ Tony Redmond, ${ }^{3}$ David F. Garway-Heath, ${ }^{1}$ Margarita B. Zlatkova, ${ }^{2}$ \\ and Roger S. Anderson ${ }^{1,2}$
}

${ }^{1}$ National Institute for Health Research (NIHR) Biomedical Research Centre at Moorfields Eye Hospital NHS Foundation Trust and UCL Institute of Ophthalmology, London, United Kingdom

${ }^{2}$ Vision Science Research Group, School of Biomedical Sciences, University of Ulster, Coleraine, Northern Ireland, United Kingdom ${ }^{3}$ School of Optometry and Vision Sciences, Cardiff University, Cardiff, United Kingdom

Correspondence: Pádraig J. Mulholland, Moorfields Eye Hospital, 162 City Road, London EC1V 2PD, UK; padraig.mulholland@moorfields.nhs. uk.

Submitted: March 20, 2015

Accepted: August 10, 2015

Citation: Mulholland PJ, Redmond T, Garway-Heath DF, Zlatkova MB, Anderson RS. Spatiotemporal summation of perimetric stimuli in early glaucoma. Invest Ophthalmol Vis Sci. 2015;56:6473-6482. DOI:10.1167/ iovs.15-16921
Purpose. To investigate achromatic temporal summation under the conditions of standard automated perimetry (SAP), using a Goldmann III (GIII) stimulus and a stimulus scaled to the local area of complete spatial summation (Ricco's area) in open-angle glaucoma (OAG) patients and healthy age-similar control participants.

Methods. Twenty patients with OAG (mean age, 63 years; mean MD, $-3.3 \mathrm{~dB}$ ) and 15 healthy controls (mean age, 64 years) were recruited. Contrast thresholds were measured for seven stimulus durations (1-24 frames, $1.8-191.9 \mathrm{~ms})$ using a near-GIII stimulus $\left(0.48^{\circ}\right.$ diameter) and stimuli scaled to the local Ricco's area, in four oblique meridians at $8.8^{\circ}$ eccentricity in the visual field. The upper limit of complete temporal summation (critical duration) was estimated using iterative two-phase regression analysis.

Results. Median critical duration values were significantly longer $(P<0.05)$ in the OAG group for the near-GIII (107.2 ms; interquartile range [IQR], 38.0-190.5) and Ricco's area-scaled (83.2 ms, 41.7-151.4) stimuli, compared to those in healthy subjects (near-GIII, $34.7 \mathrm{~ms}$; 18.2-47.9; Ricco's area-scaled, $49.0 \mathrm{~ms} ; 25.1-64.6$ ). The greatest difference in contrast thresholds between healthy and OAG subjects (i.e., disease signal) was found when stimuli were scaled to Ricco's area and shorter than or equal to the critical duration in healthy observers.

Conclusions. Temporal summation is altered in glaucoma. The stimulus duration and area of conventional SAP may be suboptimal for identifying early functional damage. Simultaneously modulating stimulus duration, area, and luminance during the examination may improve the diagnostic capability of SAP and expand the dynamic range of current instruments.

Keywords: temporal summation, spatial summation, perimetry, glaucoma
$S_{\mathrm{d}}^{\mathrm{ta}}$ tandard automated perimetry (SAP) is central to the $\checkmark$ diagnosis and monitoring of functional deficits in glaucoma. Although widely used, SAP suffers from poor sensitivity in early glaucoma,${ }^{1}$ high variability in moderate disease,${ }^{2-4}$ and the inability to adequately monitor advanced glaucoma. ${ }^{5}$ Current test strategies typically measure contrast thresholds at predefined locations in the central visual field using achromatic stimuli of constant area and duration. The selection of such stimulus parameters was made with reference to the assumed characteristics of temporal processing in the healthy and diseased visual system, ${ }^{6-8}$ or, in the case of stimulus area, directly imported from earlier kinetic instruments.

Temporal and spatial summation have a central role in determining thresholds for perimetric stimuli. ${ }^{9}$ Ricco's law $^{10}$ states that at threshold, stimulus area and luminance are inversely related, for a range of small-area stimuli. The largest stimulus area for which Ricco's law holds is termed the area of complete spatial summation or Ricco's area. The size of Ricco's area can vary with factors, such as background luminance, ${ }^{11-13}$ visual field eccentricity, ${ }^{14}$ and some visual pathway diseases. ${ }^{15-17}$ Importantly, Ricco's area is altered in glaucoma. Fellman et al. ${ }^{17}$ reported a disproportionately larger difference in contrast thresholds for small than for large stimuli, between patients with glaucoma and healthy controls; a difference that was attributed to changes in spatial summation. More recent work by Redmond et al. ${ }^{16}$ found Ricco's area to be larger in early glaucoma than in healthy eyes, entirely accounting for the disproportionate differences in threshold for a range of stimulus areas. Others report measurement variability to be reduced in glaucoma subjects with a Goldmann V stimulus when compared to a Goldmann III (GIII) stimulus (Gardiner S, et al. IOVS 2013; 54:ARVO E-Abstract 2636). ${ }^{18}$ This difference has been attributed to variations in retinal ganglion cell (RGC) number and, it may be hypothesized, the degree of spatial summation exhibited for each stimulus. If this were the case, it would seem appropriate to vary stimulus area, in addition to luminance, to improve the sensitivity and repeatability of SAP. ${ }^{16}$

In the temporal domain, summation is described by Bloch's law. ${ }^{19}$ This is analogous to Ricco's law, but with stimulus duration and luminance displaying a reciprocal relationship at threshold for short-duration stimuli. The longest duration for 
which Bloch's law holds is termed Bloch's duration, or more commonly, the critical duration. Classical studies have reported the critical duration to be in the range of $100 \mathrm{~ms}$; this varying with factors, such as stimulus area and background luminance. ${ }^{11}$ Based upon these estimates, in addition to considering the assumed minimum latency of voluntary eye movements ( $\sim 250 \mathrm{~ms})$, a stimulus duration in the region of 100 to $200 \mathrm{~ms}$ was chosen for SAP. ${ }^{6,20}$ This choice was based on the assumption that at this duration, the summation limit is reached (i.e., no further summation occurs) and, thus, contrast threshold is independent of stimulus duration. However, recent work has cast doubt on these assumptions. We have found the critical duration in healthy subjects for an achromatic near-GIII stimulus to be approximately $30 \mathrm{~ms}$ at $8.8^{\circ}$ eccentricity in the visual field, with partial summation evident for stimuli in the range of 30 to $198.3 \mathrm{~ms}^{8}{ }^{8}$ Furthermore, considering the rationale for the choice of a $200 \mathrm{~ms}$ stimulus in SAP, if temporal summation is disturbed early in glaucoma, it may be that this choice of stimulus actually inhibits the ability of SAP to identify early change.

A number of studies have examined the influence of glaucoma on temporal summation. Dannheim and Drance ${ }^{21}$ generated temporal summation functions in regions of relative scotoma and perimetrically normal areas of the visual field in glaucoma patients. They report contrast thresholds to be elevated for all stimulus durations presented in defective locations, but the shape of the summation function to be unaffected by glaucomatous RGC loss. Other studies have estimated the critical duration for achromatic spot stimuli using a range of experimental methodologies and found it to remain unchanged in glaucoma patients compared to healthy control subjects. ${ }^{22-24}$ Several investigators, however, present evidence in opposition of such findings. Holmin and $\mathrm{Krakau}^{25}$ reported contrast thresholds for short duration stimuli to be elevated disproportionately to those for long duration stimuli in glaucoma subjects, with respect to healthy controls. This change was later attributed to a rarefaction of "channels" in glaucoma, with temporal summation increasing as part of an adaptive response in the visual system to maintain visual sensitivity. ${ }^{26}$ Hnik et al. ${ }^{27}$ also found sensitivity to high-pass resolution stimuli to be depressed to a greater extent for stimuli of short duration than for those of long duration, in the clinically and perimetrically normal eye of unilateral glaucoma patients with respect to healthy controls.

In determining the optimum parameters for perimetric stimuli, it is clear that a careful examination of temporal summation in glaucoma, and in particular the critical duration, is required. Given the intrinsic link between temporal and spatial summation, ${ }^{11,28,29}$ and considering spatial summation is greater in glaucoma than in healthy controls for a stimulus of constant duration, ${ }^{16,17}$ it may be hypothesized that temporal summation also is different in glaucoma. To date, to our knowledge this hypothesis has not been tested under the conditions of SAP with a GIII stimulus, or a stimulus scaled to reflect localized spatial processing (i.e., spatiotemporal summation). As a result, it is unclear as to what stimulus duration is optimum for use in SAP when performed with a standard GIII stimulus, or a stimulus scaled for localized spatial processing.

The purpose of this study was to determine if temporal summation is altered in early glaucoma when examined using a standard GIII stimulus, and study the effect on temporal summation measurements of scaling a stimulus to the local Ricco's area in glaucoma patients and healthy age-similar control participants.

\section{Methods}

\section{Participants}

Twenty participants with open-angle glaucoma (OAG; mean age, 63.1 years; range, $48-80$ years) and 15 healthy control participants (mean age, 64.1 years; range, 40-81 years) were recruited for this study. Healthy control participants were examined as part the accompanying study, ${ }^{30}$ with the same inclusion and exclusion criteria applied here. Healthy participants were recruited and tested at Moorfields Eye Hospital (MEH; London, UK, $n=12$ ) or the University of Ulster, Coleraine (UUC, $n=3$ ). All healthy participants had a visual acuity of $20 / 30(6 / 9)$ or better in the test eye, normal IOP $(\geq 11$ and $\leq 21 \mathrm{~mm} \mathrm{Hg}$ ), and optical coherence tomograph (OCT) peripapillary retinal nerve fiber layer (RNFL) within normal limits (Spectralis; Heidelberg Engineering GmbH, Heidelberg, Germany). Glaucoma patients were recruited and tested at MEH. Of the patients recruited, 19 had been diagnosed previously with primary OAG, and one with pigment dispersion glaucoma. All glaucoma patients had a corrected visual acuity of 20/30 (6/9) or better in the test eye. Spherical refractive error was within \pm 6.00 diopter sphere (DS) in any meridian with astigmatism less than 3.00 diopter cylinder (DC). There were no significant media opacities or concurrent ocular pathology in any subject, as determined by slit-lamp assessment of the anterior eye and dilated biomicroscopic assessment of the posterior segment. Spectralis OCT peripapillary RNFL thickness was outside normal limits in at least one sector in all patients. Visual fields were examined using the 242 SITA standard test on the Humphrey visual field analyzer (Carl Zeiss Meditec, Dublin, CA, USA). Mean MD was -3.33 dB (range, -0.12 to -12.2 ) and mean PSD was $4.80 \mathrm{~dB}$ (range, 1.59-14.2 dB).

Ethical approval for this work was given by the LondonCentral National Research Ethics Service committee and the University of Ulster Biomedical Sciences Research Ethics Committee. The research protocol adhered to the tenets of the Declaration of Helsinki.

\section{Apparatus and Stimuli}

Stimuli were presented on a $\gamma$-corrected 21-inch Phillips FIMI MGD-403 achromatic CRT monitor (pixel resolution of $976 \times$ 1028, frame rate $121 \mathrm{~Hz}$, MEH site; Ampronix, Irvine, CA, USA) or a $\gamma$-corrected 21-inch Sony GSM F500-PST CRT monitor (pixel resolution of $640 \times 480$, frame rate $120 \mathrm{~Hz}$, UUC site; Sony Corp., Tokyo, Japan) using a ViSaGe MKII stimulus generator (Cambridge Research Systems, Rochester, UK) and the CRS toolbox (version 1.27) for MATLAB (version R2011a; The MathWorks, Inc., Natick, MA, USA). A background luminance of $10 \mathrm{~cd} / \mathrm{m}^{2}$ was used for all tests. Before the start of each experiment the CRT display was permitted a 1-hour warm-up time.

Data collection in this study was split into two phases. In the first phase, an area-threshold that was intended to be smaller than Ricco's area by a defined amount $\left(0.20 \log \mathrm{deg}^{2}\right)$ in each subject was generated for test locations at $8.8^{\circ}$ eccentricity along the $45^{\circ}, 135^{\circ}, 225^{\circ}$, and $315^{\circ}$ meridians of the visual field. To achieve this, circular stimuli of fixed duration $(191.9 \mathrm{~ms})$ and luminance $\left(\Delta \mathrm{I}=5.3 \mathrm{~cd} / \mathrm{m}^{2}, \log \Delta \mathrm{I} / \mathrm{I}=\right.$ $-0.3)$ but varying in area, were presented. Stimulus area was modulated in line with subject responses to estimate threshold area. It was assumed that the area of this threshold stimulus was slightly smaller than Ricco's area in both groups since the luminance used was greater than the approximate threshold expected for a stimulus equal to Ricco's area $\left(\Delta \mathrm{I} 3.3 \mathrm{~cd} / \mathrm{m}^{2}, \log \right.$ $\Delta \mathrm{I} / \mathrm{I}-0.5$; Mulholland et al. IOVS 2013;54:ARVO E-Abstract 


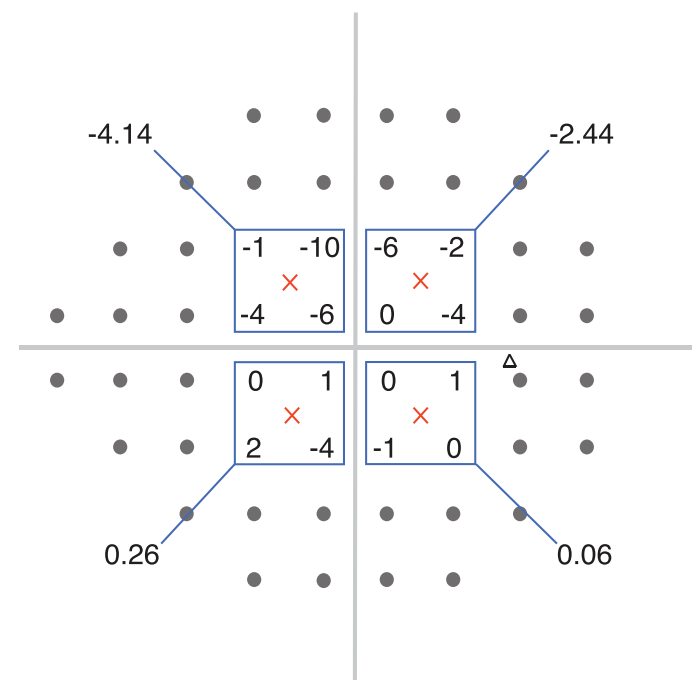

Figure 1. Test locations used in the current study (red crosses) superimposed on a 24-2 Humphrey test grid (black spots). Pointwise TD values were estimated for each test location as the mean of the four surrounding test points on HFA test grid. Numbers shown are for illustration purposes.

3924). ${ }^{14,16,31}$ As the average spatial summation function for glaucoma patients was simply translated across the size-axis, when compared to healthy age-similar control subjects, ${ }^{16}$ an area-modulation paradigm with a stimulus of constant luminance was deemed to be an appropriate and efficient method to gain an estimate of Ricco's area for the purposes of this study. A stimulus smaller than Ricco's area was desired to ensure that complete spatial summation was exhibited for all stimulus presentation durations in this study (phase 2).

In the second phase of the experiment, achromatic temporal summation functions were measured at the same test locations using a near-GIII stimulus $\left(0.48^{\circ}\right.$ diameter $)$ and the Ricco's area-scaled stimuli from phase one. For healthy observers, contrast thresholds collected for a near-GIII stimulus in the accompanying study ${ }^{30}$ were used. To construct each summation function, contrast thresholds were measured for seven stimuli of different duration (1-24 frames, 1.8-191.9 ms) within separate test runs. The order of the stimuli tested (i.e., duration and area combinations) was chosen at random. Regular rest periods were provided at intervals throughout each data collection session and when requested by the subject.

\section{Psychophysical Procedure}

Area (phase one) and contrast (phase two) thresholds were estimated using a randomly interleaved $1 / 1$ staircase with a Yes/No response paradigm. Each staircase terminated after six reversals, with threshold being calculated as the mean of the final four reversal values. In phase one, stimulus area was varied by $10 \%$ up to 3 reversals. Once 3 reversals were exceeded, area was varied by $5 \%$. In phase 2 , stimulus contrast varied by $0.05 \log$ units $(0.5 \mathrm{~dB})$, following each response. The false-positive rate was monitored through the presentation of stimuli with $0 \%$ contrast. If the false-positive rate was greater than $20 \%$ in any run, those data were discarded and the test run was repeated. Before undertaking each phase of the experiment, a trial run was provided to each participant. Only when it was clear that the subject understood the test fully were study measurements performed.

\section{Data Analysis}

Area-Modulation Data. For the purposes of statistical analysis, mean threshold area values (Ricco's area estimates) were calculated for the superior and inferior hemifield in each subject. ${ }^{16}$ A Mann-Whitney $U$ test then was used to examine for statistically significant differences in area thresholds between glaucoma patients and healthy observers in each hemifield. Individual area thresholds for glaucoma patients were also grouped according to total deviation (TD) values (from SAP) to investigate the association between spatial summation and local visual field depression. As each test location used in this study did not lie exactly on any test point on the Humphrey 24-2 (SAP) test grid, TD values were estimated as a mean of TD values for the four locations surrounding $8.8^{\circ}$ (Fig. 1). Before this calculation individual TD values were first converted to linear units $\left(\mathrm{TD}_{\mathrm{Lin}}\right.$, Equation 1), with the mean $\mathrm{TD}_{\mathrm{Lin}}$ value then converted back to $\mathrm{dB}$ units (Equation 2).

$$
\begin{gathered}
\mathrm{TD}_{\mathrm{Lin}}=10^{(\mathrm{TD} / 10)} \\
\mathrm{TD}=10^{*}\left[\log _{10}\left(\mathrm{TD}_{\mathrm{Lin}}\right)\right]
\end{gathered}
$$

Ricco's area estimates then were grouped into one of five strata $(\mathrm{TD} \geq 0,-1 \leq \mathrm{TD}<0,-2 \leq \mathrm{TD}<-1,-3 \leq \mathrm{TD}<-2$ and TD $<-3$ ). A Kruskal-Wallis test (and post hoc MannWhitney $U$ tests as appropriate) was applied to test for a statistically significant difference in threshold area with changes in localized visual field depression.

Temporal Summation Data. Contrast thresholds and stimulus durations were expressed using identical metrics to those used in the accompanying study ${ }^{30}$ (i.e., contrast energy values calculated as the product of stimulus increment luminance, duration in $\mathrm{s}$ and area in $\mathrm{deg}^{2}$, and stimulus durations as Bridgeman equivalents in $s$ incorporating known phosphor persistence values for the $\mathrm{P} 45$ phosphor incorporated in the Phillips FIMI display ${ }^{32}$ ). Should the contrast required at threshold for a given location exceed the maximum for the display monitor being used (i.e., ceiling effect), data from that location were excluded from further analysis. The critical duration was estimated for each suitable test location using iterative two-phase regression analysis. Briefly, this analysis attempts to fit two lines to the data set. The slope of the first line is constrained to zero in line with Bloch's law (constant energy at threshold), with the slope of the second line, in addition to the point at which the two constituent lines intersect (breakpoint), free to vary. The intersection point, in each case, was taken to be the critical duration estimate. If the bilinear fit failed, due to variability within the data set, or produced critical duration estimates less than the duration of the shortest stimulus ( 1 frame, $1.8 \mathrm{~ms}$ ), those data were excluded from further analysis. If a critical duration value greater than the longest stimulus duration ( 24 frames, 191.9 $\mathrm{ms})$ were estimated, a value of $191.9 \mathrm{~ms}(2.28 \mathrm{log} \mathrm{ms})$ was allocated for later analysis. The total number of successful critical duration estimates is reported in the results.

Mean critical duration values were calculated for the superior and inferior hemifield of each subject. Where data were excluded from a given location, the critical duration from the one remaining test location in the hemifield was taken to be the critical duration for that hemifield. A Mann-Whitney $U$ test was used to test for a statistically significant difference $(P$ $<0.05)$ in critical duration values between glaucoma patients and healthy observers. The association between the critical duration and localized visual field depression in glaucoma patients was investigated in a manner identical to that described above with spatial summation data (i.e., critical 


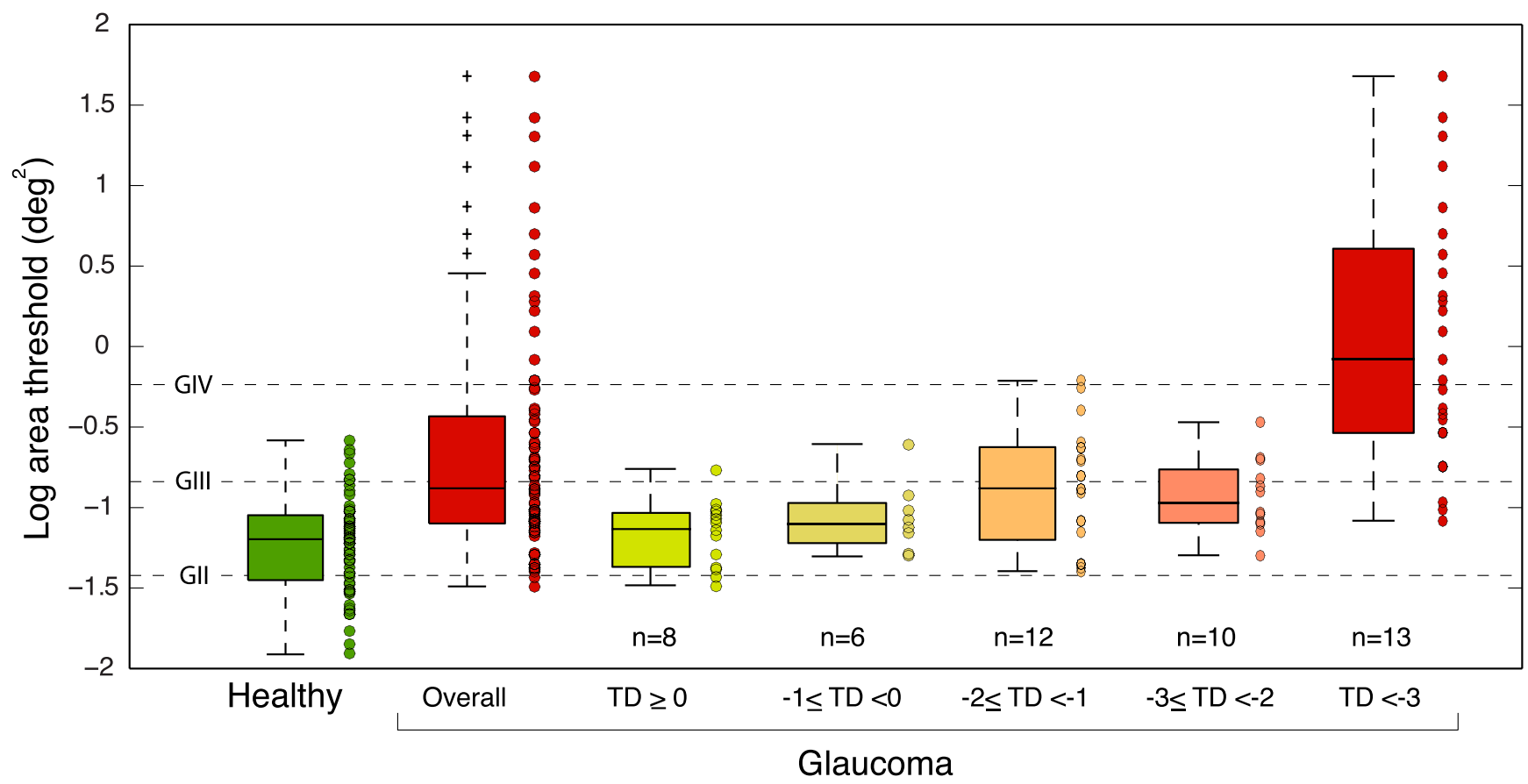

FiguRE 2. Ricco's area estimates measured using an area-modulation test grouped according to pointwise mean TD values. For reference, estimates for healthy observers (green), and all glaucoma patients (red) are included along with individual threshold values (spot markers). Boxplot outliers (defined as values outside the maximum whisker length of $q 1-1.5^{*}(q 3-q 1)$ to $q 3+1.5^{*}(q 3-q 1)$, where $q 1$ and $q 3$ are 25 th and 75 th percentiles, respectively) are represented by "+" markers. The number of patients whose data falls in each group also is listed (note that the sum of these values is greater than the total number of glaucoma patients $[n=20]$ as individual subjects can have TD values that fall within different groups).

duration values were grouped according to mean local TD values and a Kruskal-Wallis test was undertaken).

\section{RESULTS}

\section{Spatial Summation}

In agreement with previous studies, ${ }^{16,17}$ we found estimates of Ricco's area (median, interquartile range [IQR]) to be larger in glaucoma subjects (superior, $0.19 \mathrm{deg}^{2}$; IQR, 0.09-0.85; inferior, $0.10 \mathrm{deg}^{2}$; IQR, 0.08-0.20) than in healthy observers (superior, $0.06 \mathrm{deg}^{2}$; IQR, 0.04-0.08; inferior, $0.05 \mathrm{deg}^{2}$; IQR, 0.04-0.10). This difference was statistically significant (MannWhitney $U$ test; superior, $P<0.001$; inferior, $P=0.01$ ). When estimates of Ricco's area at each test location were divided into strata according to pointwise TD values in glaucoma subjects, a trend of increasing area threshold values was observed with increasing visual field depression (Fig. 2). This change was statistically significant (Kruskal-Wallis, $P<0.001$ ). Post hoc analysis with Bonferroni correction revealed a statistically significant difference $(P<0.001)$ between the TD $\geq 0$ group (median, $0.08 \mathrm{deg}^{2}$; IQR, 0.04-0.09) and the TD $<-3$ group (median, $0.83 \mathrm{deg}^{2} ;$ IQR, 0.29-3.98). Those test locations with TD values $\geq-3$ also displayed size thresholds significantly smaller than those locations with $\mathrm{TD}<-3$. There was no statistically significant difference between Ricco's area estimates in healthy observers (median, $0.06 \mathrm{deg}^{2}$; IQR, 0.04-0.09) and those from locations in glaucoma patients with TD $\geq 0(P$ $=0.33$ ).

\section{Temporal Summation}

Energy thresholds were significantly higher in the glaucoma group than in the age-similar healthy control group with the near-GIII stimulus $(P<0.001$ for stimulus durations $<191.9$ ms and $P=0.01$ for 191.9 ms duration) and Ricco's area-scaled stimuli $(P<0.001$ for all stimulus durations). Boxplots of threshold estimates for each stimulus duration, along with temporal summation functions estimated with two-phase regression analysis of across-subject median values, are shown in Figure 3. In these plots, it can be seen that the critical duration is longer in the glaucoma group, with the near-GIII and Ricco's area scaled stimuli. When thresholds were expressed in terms of luminance, the contrast at threshold $(\log \Delta \mathrm{I} / \mathrm{I})$ for a target scaled to Ricco's area and duration 24 frames (191.9 ms), was similar in the healthy (median, -0.24 ; IQR, -0.31 to -0.14 ) and glaucoma groups (median, -0.24 ; IQR, -0.34 to -0.19 ), as would be expected from the results of Redmond et al. ${ }^{16}$ For the shortest duration stimulus (1 frame, $1.8 \mathrm{~ms}$ ) luminance contrast thresholds were comparatively higher (i.e., reduced sensitivity) in the glaucoma group (median, 0.96; IQR, 0.74-1.13) relative to healthy controls (median, 0.70; IQR, 0.61-0.84) for the scaled stimulus.

A total of 140 local temporal summation measurements (60 in healthy observers, 80 in glaucoma subjects) was made using the near-GIII and Ricco's area scaled stimuli. In healthy observers, 58 temporal summation data sets measured with the near-GIII stimulus, and 57 with the Ricco's area scaled stimuli, were successfully fitted. In glaucoma subjects, 57 and 70 temporal summation functions were determined for data collected with near-GIII and Ricco's area scaled stimuli, respectively. The median critical duration was significantly longer in the glaucoma group for the near-GIII (superior $[P=$ 0.01], $77.6 \mathrm{~ms}$; IQR, 32.4-158.5; inferior [ $P<0.001], 104.7$ $\mathrm{ms}$; IQR, 77.6-162.2) and Ricco's area scaled stimuli (superior $[P=0.001], 93.3 \mathrm{~ms}$; IQR, 56.2-123.0; inferior $[P=0.007]$, $89.1 \mathrm{~ms}$; IQR, 66.1-158.5) compared to that in healthy subjects (GIII superior, $35.4 \mathrm{~ms}$; IQR, 21.4-48.9; inferior, $34.6 \mathrm{~ms}$; IQR, 19.5-47.9; Ricco's area scaled superior $33.1 \mathrm{~ms}$; IQR, 23.449.0; inferior, $53.7 \mathrm{~ms}$; IQR, 30.2-72.4). 

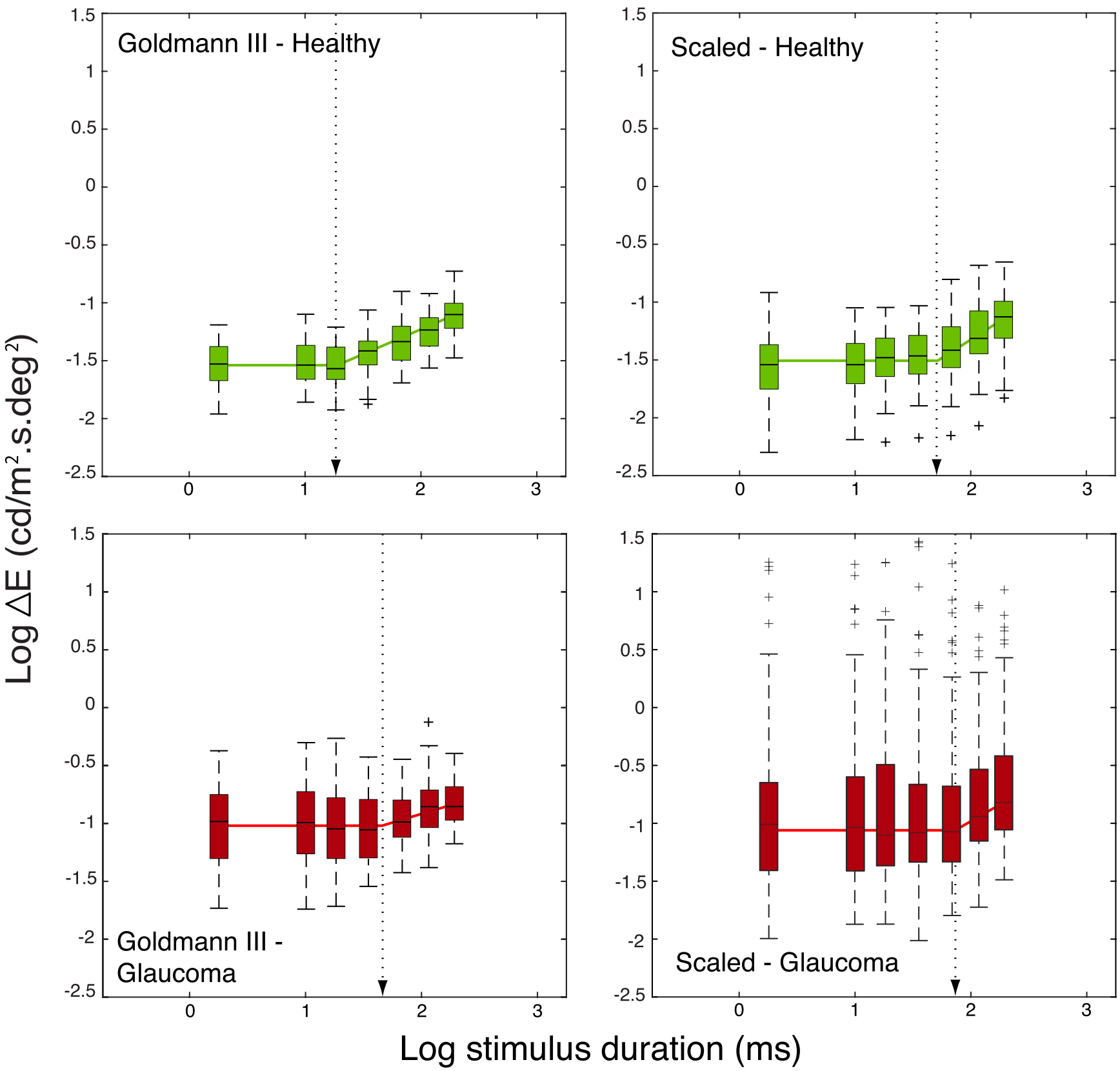

Figure 3. Thresholds values collected for individual stimulus durations using a near-GIII stimulus (left panel) and stimuli scaled to the local Ricco's area (right panel) in healthy controls (green, top) and glaucoma patients (red, bottom). Outliers for each boxplot are represented by "+" markers. Temporal summation curves, fitted to median threshold values, also are included. The arrow to the $x$-axis represents the estimated critical duration value.

Individual critical duration values grouped according to local TD values can be seen in Figure 4. A statistically significant difference was observed between the critical durations in the healthy control group and those estimated at locations in glaucoma patients with TD values greater than or equal to $0 \mathrm{~dB}$ for the near-GIII stimulus $(P=0.02)$. No statistically significant difference was observed when critical duration values for the Ricco's area scaled stimulus were compared in the healthy and glaucoma TD $\geq 0 \mathrm{~dB}$ groups $(P=$ $0.13)$. All other strata with a mean TD less than or equal to -1 $\mathrm{dB}$ displayed critical duration values that were statistically significantly longer than those in healthy observers, when a near-GIII stimulus was used (all $P \leq 0.05$ ). A statistically significant increase in the critical duration also was observed in glaucoma patients at locations with TD values $-1 \leq \mathrm{TD}<0$ ( $P$ $=0.002),-3 \leq \mathrm{TD}<-2(P=0.02)$, and $<-3(P<0.001)$ for Ricco's area scaled stimuli relative to estimates in healthy controls. Despite the median critical duration for the $-2 \leq$ TD $<-1$ group being higher than the healthy control group the difference between these strata did not reach statistical significance for the scaled stimulus $(P=0.21)$. Among glaucoma patients, an increase in the critical duration is evident between locations with TD values $\geq 0 \mathrm{~dB}$ (GIII, 70.8 ms; IQR, 30.9-190.5; Ricco's area scaled, $55.0 \mathrm{~ms}$; IQR, 34.7100.0) and $<-3 \mathrm{~dB}$ (GIII, 151.4 ms; IQR, 75.9-186.2; Ricco's area scaled, $128.8 \mathrm{~ms}$; IQR, 74.1-182.0). This difference, although present for both stimuli used, failed to reach statistical significance over the range of TD values in this 


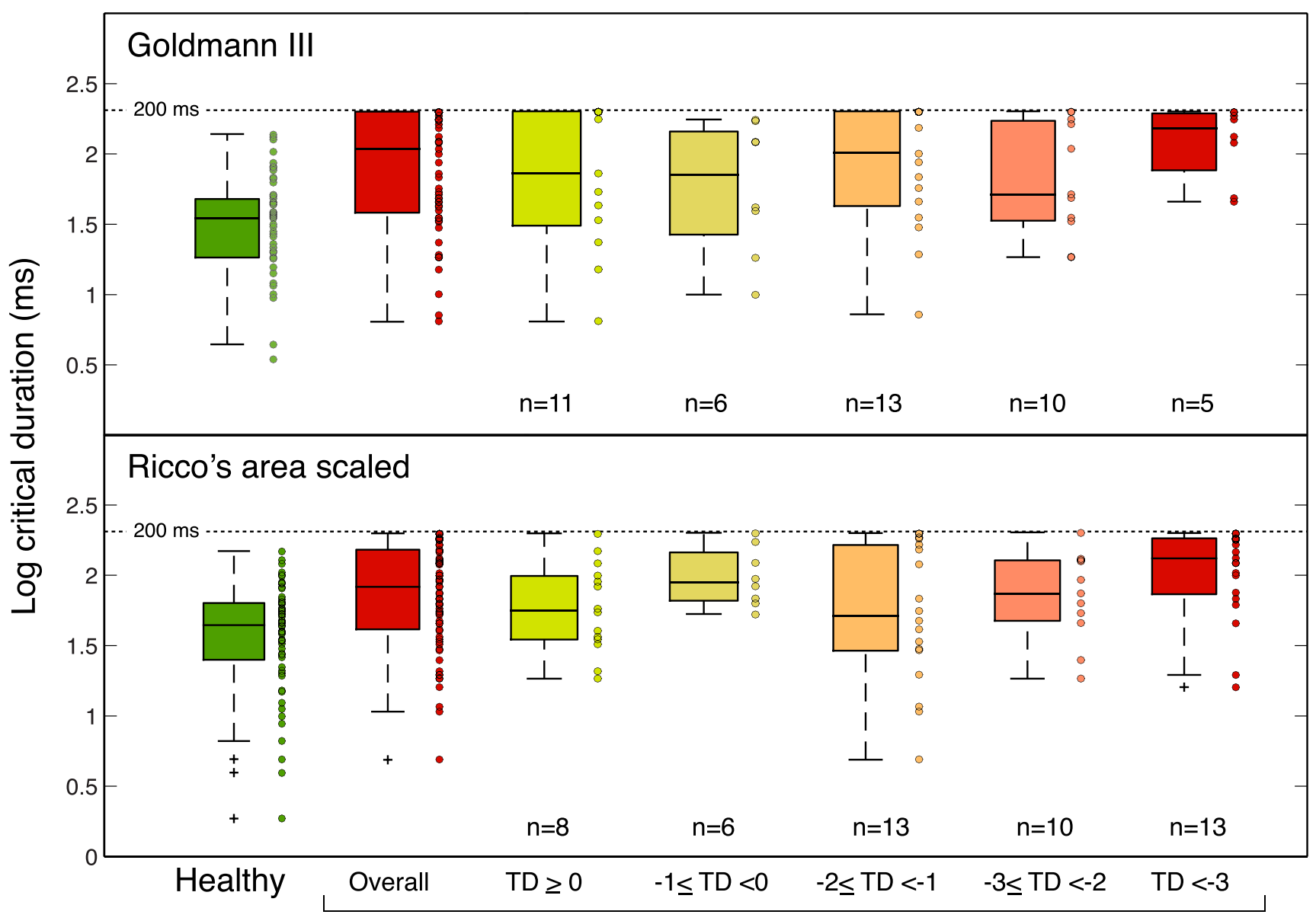

\section{Glaucoma}

Figure 4. Critical duration estimates in glaucoma patients, grouped according to local mean TD values. Included are boxplots for each group (outliers represented with "+") along with individual critical duration estimates. The number of individual subjects whose data are included in each boxplot also is listed. For reference, boxplots for the healthy group (green) and whole glaucoma group (red) are included.

study (Kruskal-Wallis, near-GIII, $P=0.49$; scaled stimulus, $P=$ $0.09)$.

\section{Discussion}

To our knowledge, this is the first study demonstrating an increase in the critical duration in glaucoma. Statistically significant increases were found when measured with a nearGIII stimulus $\left(0.48^{\circ}\right.$ diameter) and a stimulus scaled according to local spatial summation, under the same background and other stimulus conditions as used in SAP. In accordance with previous studies, ${ }^{16,17}$ we confirm Ricco's area, estimated through an area-modulation test for a stimulus of constant luminance and duration $191.9 \mathrm{~ms}$ (24 frames), is larger in patients with early glaucoma.

The results of this study appear to contradict those reported in earlier work investigating temporal summation using similar achromatic stimuli. ${ }^{21-23}$ However, variations in experimental methodology, test equipment and, perhaps most importantly, analysis techniques used to estimate the critical duration, confound comparison. For example, Dannheim and Drance ${ }^{21}$ used a stimulus $\left(0.75^{\circ}\right.$ diameter $)$ much larger than most stimuli used in our experiment. In the same study, "control" data were collected from glaucoma subjects, in regions of the visual field where no defect was apparent with either kinetic or static profile perimetry. Other studies have used analysis methods that potentially can overestimate the critical duration. ${ }^{8}$ For example, Funkhouser and Fankhauser ${ }^{22}$ and Ogawa et al. ${ }^{23}$ estimated the critical duration by fitting a bilinear function that assumed complete and then no summation. As a result, it is possible that small changes in the critical duration may have been overlooked in these studies. ${ }^{8}$ In the current study, it was only assumed that there is a duration below which complete summation took place; the slope and intercept of the second line, together with the breakpoint, in the bilinear fit were free to vary.

The effect of glaucoma on temporal summation also may be seen if one considers the threshold contrast energy values for stimuli of varying duration. When Bloch's law is satisfied, and summation complete, energy at threshold remains constant. Thus, should the critical duration increase, the range of stimulus durations for which energy remains constant at threshold would also be expected to extend. This is seen in Figure 3 where complete summation is evident for a larger range of stimulus durations in glaucoma patients compared to healthy control subjects. This result supports the findings of Homlin and $\mathrm{Krakau}^{25}$ and Hnik et al. ${ }^{27}$ It also is interesting to note that when temporal summation is complete, contrast energy at threshold does not appear to alter greatly with changes in stimulus area for either the healthy $(\log \Delta \mathrm{E}=$ $\sim-1.50$ ) or glaucoma (log $\Delta \mathrm{E}=\sim-1.00$ ) groups (Fig. 3). 


\section{Physiological Source of Temporal Summation Changes in Glaucoma}

The results of this study pose important questions about the nature of visual processing in glaucoma: Why does summation increase, and what is the physiological source of this change? To answer such questions tentatively, one must first consider summation in the healthy visual system and the various factors that can influence the critical duration and extent of Ricco's area. For example, Ricco's area and critical duration can vary with background illumination ${ }^{11,13}$ and visual field locus (Ref. 14 and Mulholland P, et al. IOVS 2013;54:ARVO E-Abstract 3924). It may be argued that such changes occur to maintain a constant signal-to-noise ratio, and thus constant sensitivity or detectability (d'), in a range of visual environments. ${ }^{11}$ In glaucoma, such changes also might occur to maintain d' at a constant level, to compensate for RGC loss, at the expense of spatial and temporal resolution. This hypothesis has been put forward previously by $\mathrm{Krakau}^{26}$ and Redmond et al. ${ }^{16}$ as a possible explanation for increasing summation in glaucoma. $\mathrm{Krakau}^{26}$ proposed that an increase in temporal summation might be explained by a reduction in the number of RGCs or available "channels." Redmond et al. ${ }^{16}$ suggested Ricco's area might enlarge to boost the signal being delivered to higher visual centers with the result that d' remains constant.

The physiological basis of the critical duration remains a matter of significant debate in published literature. It is likely that photoreceptors, ${ }^{33-35} \mathrm{RGCs}^{36,37}$ and higher visual areas ${ }^{15,38}$ each have a role, either independently or together with other structures, ${ }^{39}$ in determining the critical duration. In the case of glaucoma, it generally is accepted that RGCs are the primary cells affected in the disease. ${ }^{40,41}$ Ricco's area has been found to be larger in individuals with glaucomatous damage, possibly occurring through either an active reorganization of retinal or other neurons of the visual pathway leading to a wider convergence of RGCs on cortical cells, ${ }^{42}$ or detection becoming mediated by already present cortical receptive fields/filters of greater spatial extent to maintain input from a constant number of RGCs in the absence of any neural reorganization. ${ }^{16,31,43}$ Given the intrinsic link between spatial and temporal summation, ${ }^{11,28,29}$ and considering spatial summation changes in glaucoma, ${ }^{16,17}$ it is entirely possible that temporal summation also will be affected. Interestingly, we also observed a statistically significant increase in the critical duration in glaucoma patients relative to healthy controls when examined using stimuli scaled to reflect localized spatial summation. Here, we might expect the functional consequences of changes in RGC density to be, at least in part, compensated for through scaling stimulus area. ${ }^{16}$ Considering this, it is possible that RGC function is disturbed before, or in tandem with, structural damage in optic neuropathy, ${ }^{44}$ leading to changes in temporal summation over and above those explained by a change in spatial summation. It has been shown in animal models that before apoptotic cell death, RGCs undergo shrinkage ${ }^{45,46}$ with a reduction in dendritic arborisations, ${ }^{47}$ resulting in reduced synaptic integrity. ${ }^{48}$ Functional anomalies have been attributed to such changes. ${ }^{48,49}$ On this basis, it is reasonable to hypothesize that premorbid dysfunction in RGCs, ${ }^{44}$ in addition to cell death, may partially explain our findings.

Although generally thought of as a disease altering RGC function and density, glaucoma is increasingly considered a neurodegenerative disorder influencing the entire visual pathway, including the structure ${ }^{50-55}$ and function ${ }^{56}$ of higher visual centers. With this in mind, and considering that the visual cortex has a central role in governing temporal summation, ${ }^{15,33,38}$ it is possible that changes in the visual cortex as a consequence of glaucoma also might lead to the changes in temporal summation observed in this study. Wilson $^{15}$ found temporal summation to be disrupted in subjects with defects affecting only the visual pathway posterior to the lateral geniculate nucleus (LGN). It also has been suggested that changes in the extent of Ricco's area in glaucoma may be related to the spatial tuning of second stage cortical filters. ${ }^{31}$ Redmond et al. ${ }^{16}$ hypothesized that an enlargement of Ricco's area is the result of detection becoming mediated by alternative cortical channels, in an attempt to preserve a constant signal-to-noise ratio in the absence of structural reorganization. Specifically, they hypothesized that the largest area over which spatial summation remains linear equates to a cortical filter receiving input from 31 RGCs. As RGCs are lost in glaucoma, the area over which 31 RGCs are dispersed is larger. A cortical filter with a spatial extent equivalent to this larger area would have displayed probability summation when the RGC density was normal, but now demonstrates linear summation. Thus, Ricco's area appears to enlarge (i.e., a constant number of RGCs underlie the "perceptive" field ${ }^{31,43}$ ). In a similar manner, a cortical "detector" or filter with a long time constant may facilitate the detection of perimetric stimuli in glaucoma, and, thus, also lead to an increase in the critical duration relative to healthy observers with no active remodeling of the visual pathway. It also could be argued that second-stage cortical filters, with a specific time constant and spatial tuning, determine the size of Ricco's area and/or the critical duration in healthy subjects and glaucoma patients. In turn, such filters may function to maintain constant detectability across the visual field in a range of visual environments or in disease. Equally, it is possible that an active reorganization of the visual cortex takes place in glaucoma resulting in filters with spatiotemporal properties different to those present in the healthy visual cortex. Such neural plasticity has been observed in in vitro ${ }^{57}$ and in vivo ${ }^{58,59}$ animal studies.

\section{Variations in Spatial Summation With Stimulus Duration}

In this study, we observed a statistically significant increase in the critical duration in glaucoma patients relative to healthy controls when examined using a stimulus scaled to the localized Ricco's area. However, it must be noted that a stimulus scaled to approximate Ricco's area for a 24-frame reference presentation duration (191.9 ms) was used to construct temporal summation functions. Ricco's area is known to increase with a reduction in stimulus duration ${ }^{29}$ and, as such, the scaled stimulus used is unlikely to accurately reflect the extent of Ricco's area for all stimulus presentation durations in this study. It also is currently unknown if the effect of stimulus duration on the size of Ricco's area is different in glaucoma subjects when compared to healthy controls. Should the rate of change in Ricco's area with stimulus duration be greater in glaucoma observers, the discrepancy between the true Ricco's area and the stimulus area used in this study for stimulus durations less than 191.9 ms may be greater in this group relative to healthy controls, with the result that an artifactual change in temporal summation is observed. Further work, outside of the scope of this study, is required to firmly establish the rate of change in Ricco's area with stimulus duration in healthy subjects and glaucoma patients.

\section{Implications for Perimetry}

It is clear, from the findings presented here, that the use of a stimulus presentation time in the range of 100 to $-200 \mathrm{~ms}$ is inappropriate if one wishes to detect early disease using SAP. In Figure 3, it can be seen that the temporal summation functions 


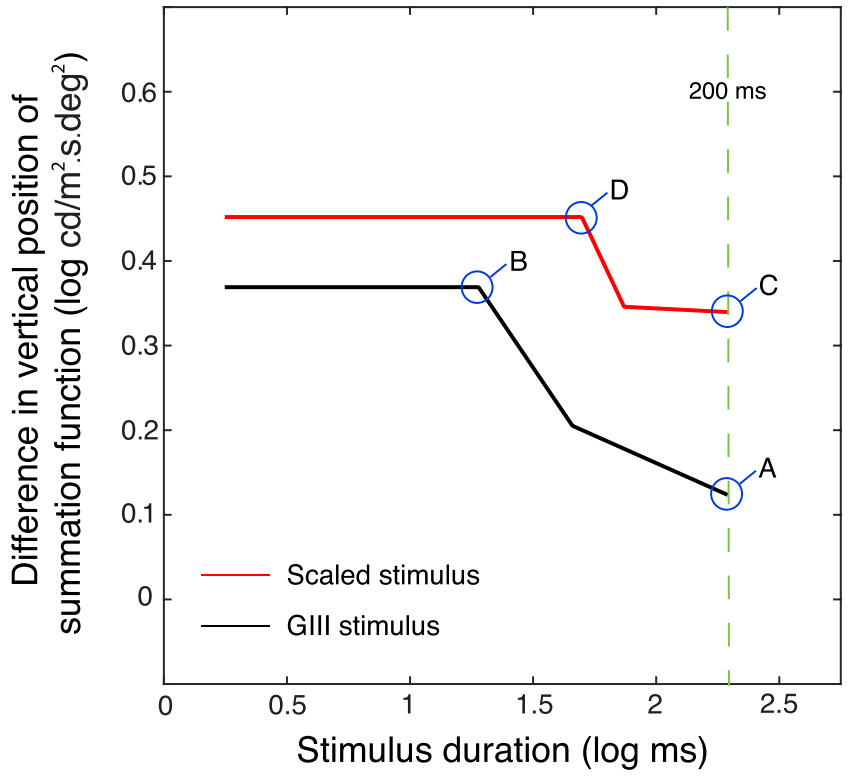

FiguRE 5. Difference in threshold contrast energy between glaucoma patients and healthy control subjects for stimuli of varying duration as predicted by 2-phase regression analysis. Data are presented for a GIII stimulus $\left(0.48^{\circ}\right.$, black $)$ and scaled stimuli (red). Labels indicate threshold differences for $A$, GIII stimulus/200 ms; $B$, GIII stimulus/ equal to critical duration in healthy subjects; $C$, scaled stimulus/200 ms and $D$, scaled stimulus/equal to critical duration in healthy subjects.

for subjects with glaucoma undergo a rightward (increase in the critical duration) and upward (increase in threshold contrast energy) shift in position compared to healthy observers. Considering this, any differences in threshold (vertical difference in the position of summation function for a single stimulus of fixed duration) between healthy observers and glaucoma patients, otherwise termed the disease signal (plotted as a function of stimulus duration in Fig. 5), may be increased if a presentation time shorter than 100 to $200 \mathrm{~ms}$ is used in combination with a GIII stimulus or stimuli scaled to the localized Ricco's area. In the case of a GIII stimulus, disease signal may be boosted by approximately $200 \%$ if a stimulus equal to the critical duration in healthy observers is used (Fig. 5 , B), in place of the standard presentation duration of $200 \mathrm{~ms}$ (Fig. 5, A). An even greater boost ( 300\%) in the disease signal may be gained if a stimulus is scaled to the localized Ricco's area and presented with a duration less than or equal to the critical duration in healthy observers for that stimulus type (Fig. 5, D). Interestingly, a comparable boost in the disease signal of SAP may be gained by reducing stimulus duration to be equal to the critical duration in healthy observers when using a GIII stimulus (Fig. 5, B), or scaling stimulus area to reflect localized spatial summation when using a presentation duration of approximately $200 \mathrm{~ms}$ (Fig. 5, C). These findings clearly demonstrated that achromatic SAP stimuli modulating in either two (luminance/area, luminance/duration) or three (luminance/area/duration) dimensions also would serve to increase the dynamic range of SAP, improve the ability to detect early RGCs loss, and ultimately permit clinicians to chart subtle underlying changes in the glaucomatous visual system, provided the increase in signal is not matched with a corresponding increase in variability. Further research is required to determine whether a stimulus modulating in one, two, or three dimensions affords the largest disease signal, and if a low degree of measurement variability can be maintained with such a stimulus (i.e., to achieve a higher signal-to-noise ratio).

\section{Conclusions}

Spatial and temporal summation are greater in glaucoma than in healthy controls. Such changes may occur in an effort to maintain constant signal-to-noise ratio in regions of the visual field served by RGCs that have either been lost or whose function is compromised. The findings of this study also have implications for the optimum design of perimetric stimuli. It is likely that stimuli modulating in area, duration, or in threedimensions (area-duration-luminance), offers the advantage of extending the dynamic range of current instruments, in addition to improving the sensitivity of SAP.

\section{Acknowledgments}

Presented in part at the European Academy of Optometry and Optics annual meeting, Warsaw, Poland, May 2014, and 21st International Imaging and Visual Field Symposium, New York, New York, United States, September 2014.

Supported by a PhD studentship from the Department for Employment and Learning, Northern Ireland (PJM), and in part by the National Institute for Health Research (NIHR) Biomedical Research Centre based at Moorfields Eye Hospital NHS Foundation Trust and UCL Institute of Ophthalmology (PJM, DFG-H). DFG-H's chair at UCL is supported by funding from the International Glaucoma Association. Conference presentation of this work was supported by the Moorfields Association in the form of a travel grant (PJM). The views expressed are those of the authors and not necessarily those of the NHS, the NIHR, or the Department of Health.

Disclosure: P.J. Mulholland, Heidelberg Engineering (R); T. Redmond, Heidelberg Engineering (F); D.F. Garway-Heath, Carl Zeiss Meditec (F, R), Heidelberg Engineering (F, R), P; M.B. Zlatkova, None; R.S. Anderson, Heidelberg Engineering (F, R)

\section{References}

1. Tafreshi A, Sample PA, Liebmann JM, et al. Visual functionspecific perimetry to identify glaucomatous visual loss using three different definitions of visual field abnormality. Invest Ophthalmol Vis Sci. 2009;50:1234-1240.

2. Henson DB, Chaudry S, Artes PH, Faragher EB, Ansons A. Response variability in the visual field: comparison of optic neuritis, glaucoma, ocular hypertension, and normal eyes. Invest Ophthalmol Vis Sci. 2000;41:417-421.

3. Artes PH, Hutchison DM, Nicolela MT, LeBlanc RP, Chauhan BC. Threshold and variability properties of matrix frequencydoubling technology and standard automated perimetry in glaucoma. Invest Ophthalmol Vis Sci. 2005;46:2451-2457.

4. Russell RA, Crabb DP, Malik R, Garway-Heath DF. The relationship between variability and sensitivity in large-scale longitudinal visual field data. Invest Ophthalmol Vis Sci. 2012; 53:5985-5990.

5. Wall M, Woodward KR, Doyle CK, Artes PH. Repeatability of automated perimetry: a comparison between standard automated perimetry with stimulus size III and V, matrix, and motion perimetry. Invest Ophthalmol Vis Sci. 2009;50:974979.

6. International Council of Ophthalmology. Perimetric Standards and Perimetric Glossary of the International Council of Ophthalmology. The Hague, London: Dr. W. Junk bv Publishers; 1979.

7. Aulhorn E, Harms H. Visual perimetry. In: Jameson D, Hurvich L, eds. Visual Psychophysics. New York: Springer-Verlag; 1972: 102-145. 
8. Mulholland PJ, Redmond T, Garway-Heath DF, Zlatkova MB, Anderson RS. Estimating the critical duration for temporal summation of standard achromatic perimetric stimuli. Invest Ophthalmol Vis Sci. 2015;56:431-437.

9. Anderson RS. The psychophysics of glaucoma: improving the structure/function relationship. Prog Ret Eye Res. 2006;25:7997.

10. Ricco A. Relazione fra il minimo angolo visuale e l'intensità luminosa. Memorie della Regia Academia di Scienze, lettere ed arti in Modena. 1877;17:47-160.

11. Barlow HB. Temporal and spatial summation in human vision at different background intensities. J Physiol. 1958;141:337350 .

12. Redmond T, Zlatkova MB, Vassilev A, Garway-Heath DF, Anderson RS. Changes in Ricco's area with background luminance in the S-cone pathway. Optom Vis Sci. 2013;90: 66-74.

13. Glezer VD. The receptive fields of the retina. Vision Res. 1965; 5:497-525.

14. Wilson ME. Invariant features of spatial summation with changing locus in the visual field. J Physiol. 1970;207:611622 .

15. Wilson ME. Spatial and temporal summation in impaired regions of the visual field. J Physiol. 1967;189:189-208.

16. Redmond T, Garway-Heath DF, Zlatkova MB, Anderson RS. Sensitivity loss in early glaucoma can be mapped to an enlargement of the area of complete spatial summation. Invest Ophthalmol Vis Sci. 2010;51:6540-6548.

17. Fellman RL, Lynn JR, Starita RJ, Swanson WH. Clinical importance of spatial summation in glaucoma. In: Heijl A, ed. Perimetry Update 1988/1989. Amsterdam/Milano: Kugler \& Gedini; 1989:313-324.

18. Wall M, Doyle CK, Zamba KD, Artes P, Johnson CA. The repeatability of mean defect with size III and size V standard automated perimetry. Invest Ophthalmol Vis Sci. 2013;54: 1345-1351.

19. Bloch AM. Experiences sur la vision. C R Soc Biol (Paris). 1885;37:493-495.

20. Johnson CA. Psychophysical factors that have been applied to clinical perimetry. Vision Res. 2013;90:25-31.

21. Dannheim F, Drance SM. Psychovisual disturbances in glaucoma. A study of temporal and spatial summation. Arch Ophthalmol. 1974;91:463-468.

22. Funkhouser AT, Fankhauser F. Temporal summation measurements with the Octopus 1-2-3 perimeter. Ger J Ophthalmol. 1994;3:120-128.

23. Ogawa T, Furuno F, Seki A, Suzumura H, Yabuki K, Matsuo H. Temporal summation of normal eyes and impaired regions of the visual field. Nippon Ganka Gakkai Zasshi. 1984;88: 1216-1223.

24. Kono M, Yamade S. Temporal integration in diseased eyes. Int Ophthalmol. 1996;20:231-239.

25. Holmin C, Krakau CE. Variability of glaucomatous visual field defects in computerized perimetry. Albrecht Von Graefes Arch Klin Exp Ophthalmol. 1979;210:235-250.

26. Krakau C. Temporal summation and perimetry. Ophthalmic Res. 1989;21:49-55.

27. Hnik P, Chauhan BC, Drance SM, Chan A. Temporal Summation in Early Glaucoma. In: Wall M, Heijl A. Perimetry Update 1996/1997. Proceedings of the XIIth International Perimetric Society Meeting, Wurzburg, Germany, June 4-8, 1996;1996:223.

28. Baumgardt E. Visual spatial and temporal summation. Nature. 1959;184:1951-1952.

29. Owen W. Spatiotemporal integration in the human peripheral retina. Vision Res. 1972;12:1011-1026.
30. Mulholland PJ, Redmond T, Garway-Heath DF, Zlatkova MB, Anderson RS. The effect of age on the temporal summation of achromatic perimetric stimuli. Invest Ophthalmol Vis Sci. 2015;56:6467-6472.

31. Swanson WH, Felius J, Pan F. Perimetric defects and ganglion cell damage: interpreting linear relations using a two-stage neural model. Invest Ophthalmol Vis Sci. 2004;45:466-472.

32. Mulholland PJ, Zlatkova MB, Redmond T, Garway-Heath DF, Anderson RS. Effect of varying CRT refresh rate on the measurement of temporal summation. Ophthal Physiol Opt. 2015;35:582-590.

33. Hartline HK. A quantitative and descriptive study of the electric response to illumination of the arthropod eye. Am J Physiol. 1928;83:466-483.

34. Hood DC, Grover BG. Temporal summation of light by a vertebrate visual receptor. Science. 1974;184:1003-1005.

35. Hansen RM, Moskowitz A, Tavormina JL, Bush JN, Soni G, Fulton AB. Temporal summation in children with a history of retinopathy of prematurity (ROP). Invest Ophthalmol Vis Sci. 2015;56:914-917.

36. Granit R, Davis W. Comparative studies on the peripheral and central retina IV: temporal summation of subliminal visual stimuli and the time course of the excitory after-effect. $A m J$ Physiol. 1931;98:644-653.

37. Baumgardt E, Hillmann B. Duration and size as determinants of peripheral retinal response. J Opt Soc Am A. 1961;51:340344.

38. Swanson WH, Pan F, Lee BB. Chromatic temporal integration and retinal eccentricity: psychophysics, neurometric analysis and cortical pooling. Vision Res. 2008;48:2657-2662.

39. Battersby W, Schuckman H. The time course of temporal summation. Vision Res. 1970;10:263-273.

40. Harwerth RS, Carter-Dawson L, Shen F, Smith EL III, Crawford ML. Ganglion cell losses underlying visual field defects from experimental glaucoma. Invest Ophthalmol Vis Sci. 1999;40: 2242-2250.

41. Kendell KR, Quigley HA, Kerrigan LA, Pease ME, Quigley EN. Primary open-angle glaucoma is not associated with photoreceptor loss. Invest Ophthalmol Vis Sci. 1995;36:200-205.

42. Schefrin BE, Bieber ML, McLean R, Werner JS. The area of complete scotopic spatial summation enlarges with age. $J O p t$ Soc Am A Opt Image Sci Vis. 1998;15:340-348.

43. Vassilev A, Ivanov I, Zlatkova MB, Anderson RS. Human S-cone vision: relationship between perceptive field and ganglion cell dendritic field. J Vis. 2005;5:823-833.

44. Porciatti V, Ventura LM. Retinal ganglion cell functional plasticity and optic neuropathy: a comprehensive model. $J$ Neuro-Ophthalmol. 2012;32:354-358.

45. Morgan JE, Datta AV, Erichsen JT, Albon J, Boulton ME. Retinal ganglion cell remodelling in experimental glaucoma. Adv Exp Med Biol. 2006;572:397-402.

46. Morgan JE, Uchida H, Caprioli J. Retinal ganglion cell death in experimental glaucoma. Br J Ophthalmol. 2000;84:303-310.

47. Kisiswa L, Dervan AG, Albon J, Morgan JE, Wride MA. Retinal ganglion cell death postponed: giving apoptosis a break? Ophthalmic Res. 2010;43:61-78.

48. Weber AJ, Harman CD. Structure-function relations of parasol cells in the normal and glaucomatous primate retina. Invest Ophthalmol Vis Sci. 2005;46:3197-3207.

49. Fortune B, Bui BV, Morrison JC, et al. Selective ganglion cell functional loss in rats with experimental glaucoma. Invest Ophthalmol Vis Sci. 2004;45:1854-1862.

50. Yu L, Xie B, Yin X, et al. Reduced cortical thickness in primary open-angle glaucoma and its relationship to the retinal nerve fiber layer thickness. PLoS One. 2013;8:e73208.

51. Gupta N, Ang LC, Noel de Tilly L, Bidaisee L, Yucel YH. Human glaucoma and neural degeneration in intracranial optic nerve, 
lateral geniculate nucleus, and visual cortex. BrJ Ophthalmol. 2006;90:674-678.

52. Yücel YH, Zhang Q, Weinreb RN, Kaufman PL, Gupta N. Effects of retinal ganglion cell loss on magno-, parvo-, koniocellular pathways in the lateral geniculate nucleus and visual cortex in glaucoma. Prog Retin Eye Res. 2003;22:465-481.

53. Hernowo AT, Boucard CC, Jansonius NM, Hooymans JM, Cornelissen FW. Automated morphometry of the visual pathway in primary open-angle glaucoma. Invest Ophthalmol Vis Sci. 2011;52:2758-2766.

54. Zikou AK, Kitsos G, Tzarouchi LC, Astrakas L, Alexiou GA, Argyropoulou MI. Voxel-based morphometry and diffusion tensor imaging of the optic pathway in primary open-angle glaucoma: a preliminary study. AJNR Am J Neuroradiol. 2012; 33:128-134.

55. Williams AL, Lackey J, Wizov SS, et al. Evidence for widespread structural brain changes in glaucoma: a preliminary voxel- based MRI study. Invest Ophthalmol Vis Sci. 2013;54:58805887.

56. Duncan RO, Sample PA, Weinreb RN, Bowd C, Zangwill LM. Retinotopic organization of primary visual cortex in glaucoma: a method for comparing cortical function with damage to the optic disk. Invest Ophthalmol Vis Sci. 2007;48:733-744.

57. Kirkwood A, Lee HK, Bear MF. Co-regulation of long-term potentiation and experience-dependent synaptic plasticity in visual cortex by age and experience. Nature. 1995;375:328331.

58. Eyding D, Schweigart G, Eysel UT. Spatiotemporal plasticity of cortical receptive fields in response to repetitive visual stimulation in the adult cat. Neuroscience. 2002;112:195-215.

59. Pizzorusso T, Medini P, Berardi N, Chierzi S, Fawcett JW, Maffei L. Reactivation of ocular dominance plasticity in the adult visual cortex. Science. 2002;298:1248-1251. 